\title{
COLETA DE DADOS REALIZADA POR QUESTIONÁRIO ENVIADO PELO CORREIO: MÉTODO EFICAZ?
}

ARTIGOS

* Lucia Maria Barbosa de Oliveira **Walter Fernando Araújo de Moraes

Três casos internacionais de pesquisas realizadas com sucesso validam o uso da coleta de dados realizada por questionário enviado pelo correio.

Three international cases of research successfully carried out proves the mail questionary methods for gathering data.

PALAVRAS-CHAVE:

Questionário por correio, método de pesquisa, pesquisa pelo correio, pesquisa social, construção de questionário.

KEY WORDS:

Mail questionnaire, mail survey, survey method, social research, questionnaire design.

* Mestre em Administração pela UFMG, Ph.D. pela London School of Economics and Political Science, Professora Adjunta da UFPE, Pesquisadora do $\mathrm{CNPq}$.

**Mestre em Engenharia da Produção pela UFSC, Ph.D. pela University of Manchester Institute of Science and Technology, Professor Adjunto da UFPE

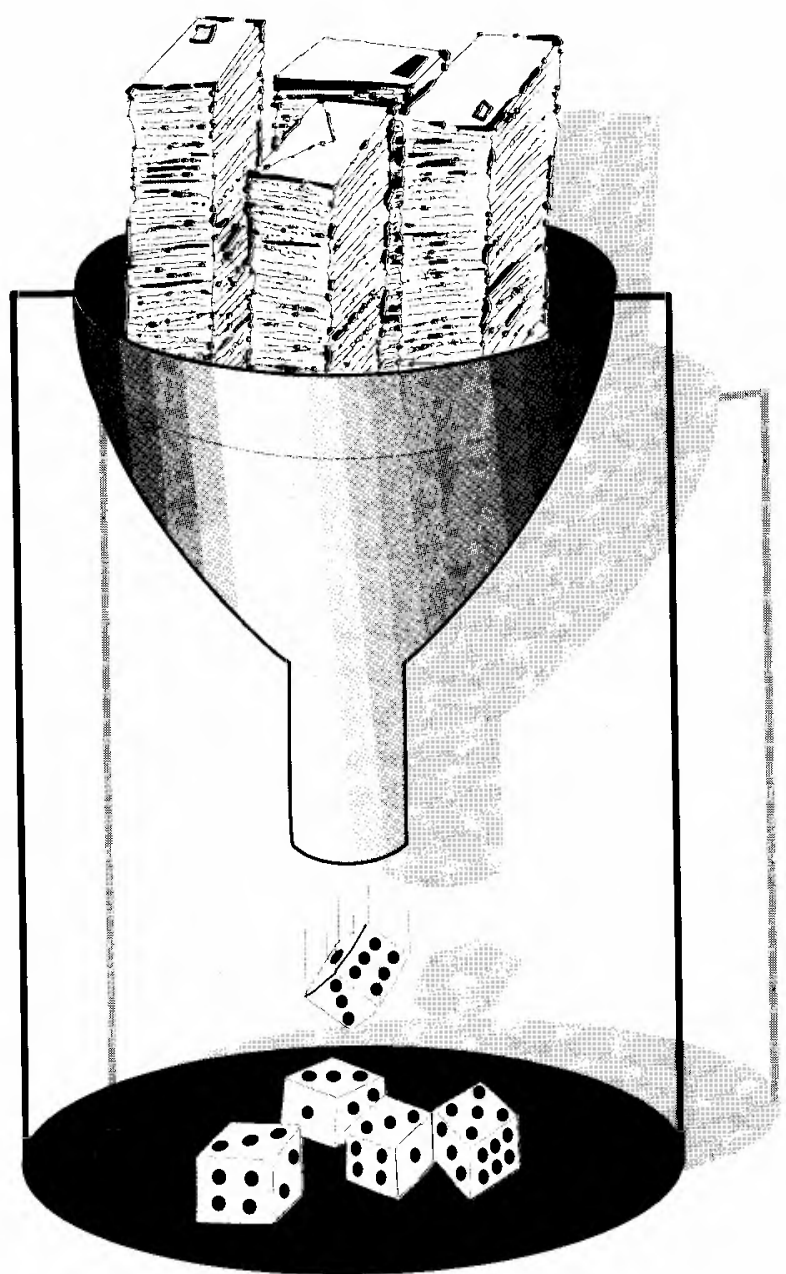

São Paulo, v. 34, n. 4, p. 85-92

Jul./Ago. 1994 
1. MINISTÉRIO DA EDUCAÇĀO E CULTURA. Relatório de cursos por IES. Brasília: Secretaria de Educaçāo Superior. novembro, 1988.

2. REVISTA EXAME. São Paulo, edição 459 , anо 22, n. 16, p.38$45,1990$.

3. MOSER, C. A. SUrvey methoos in soclal investigation. London: Heinemann, 1969 , p. 175.
A cada dia que passa, mais aumenta a necessidade de se realizar pesquisas na área de Administração. No Brasil, existem 261 cursos de Administração, de acordo com a Secretaria de Educação Superior do Ministério de Educação e Cultura, ${ }^{1}$ sendo tais cursos dos mais procurados pelos vestibulandos. Em artigo recente, a revista Exame ${ }^{2}$ destaca o despreparo dos jovens recém-diplomados em Administração no Brasil para atender às necessidades do mercado. É demonstrada a preocupação de algumas escolas mais conceituadas em adequar os currículos de tais cursos, buscando atalhos para reduzir o gap que separa a academia da vida prática.

Pesquisas precisam ser realizadas visando a analisar a realidade e descobrir as necessidades das empresas. Mas, se por um lado isso ocorre, por outro, cada vez mais o volume de verbas destinadas à pesquisa tem sido reduzido. Dessa forma, os pesquisadores, a cada dia que passa, têm que procurar métodos alternativos e menos dispendiosos para realizar seu trabalho, sem prejudicar, de forma alguma, a qualidade da pesquisa.

Entre os métodos de se $\mathrm{co}^{-}$ letar dados nas empresas, um merece atenção especial: aquele em que as informações são collidas nas organizações através de questionários enviados pelo correio. Sem dúvida, é um método atrativo, que conduz a resultados confiáveis, além de utilizar procedimentos que tornam os seus custos reduzidos. Assim, nos dias atuais em que a minimização de custos constitui matéria essencial na realização de qualquer atividade, na pesquisa não poderia ser diferente.

O método do questionário enviado pelo correio, para ser bem-sucedido, exige que alguns cuidados devam ser levados em consideração, tais como: a estrutura das questões, a apresentação, a extensão e a clareza do questionário. Neste artigo, é dada ênfase ao Total Design Method -
TDM, em que são sugeridas algumas regras básicas para se elaborar e obter sucesso na aplicação desse método nas pesquisas realizadas na área de Administração.

\section{REGRAS BÁSICAS PARA UTILIZAR O MÉTODO DE QUESTIONÁRIO REMETIDO PELO CORREIO}

O uso do método de questionário remetido pelo correio tem sido criticado em virtude da suposta dificuldade de obtenção de respostas adequadas e seguras. Mas, como afirma Moser: "embora séria, essa dificuldade nâo pode esconder os méritos do método". ${ }^{3}$

As seguintes vantagens estão associadas ao uso desse método: é mais rápido e mais econômico do que os outros métodos, principalmente quando os pesquisados estão geograficamente dispersos; permite que o pesquisado apresente respostas mais acuradas, especialmente quando são questōes que podem causar embaraço na presença de um entrevistador; se a resposta demandar a consulta de documentos, o questionário preenchido pelo pesquisado pode ser o preferido e o resultado, mais acurado, pois ele terá mais tempo para responder.

Entretanto, devem ser destacadas as seguintes limitações: é utilizado somente quando as questões são simples e diretas, com instruçôes claras e definidas; é inflexível, no sentido que não pode ser realizada uma checagem dos dados coletados; é inapropriado quando se deseja respostas espontâneas; não dá oportunidade de suplementar as respostas do pesquisado através da observação de dados.

O método em questão pode apresentar resultados compensadores desde que seja 
adequadamen-

te utilizado.

Goode e Ilatt,

por exemplo, es-

clarecem que:

"a adequação do

questionário re-

metido dependerá

das exigências do

problema da pes-

quisa em relação

ao tipo de informação necessária, ao tipo de informante alcançado, à acessibilidade dos informantes e à precisão da hipótese". ${ }^{4}$

Salienta-se, na literatura, o Total Design Method - TDM - sugerido por Dillman ${ }^{5}$ para construção e envio de questionários pelo correio.

O TDM indica os seguintes aspectos que devem ser considerados para otimizar o número de respostas:

- minimizar o custo para o respondente, tornando fácil e breve o trabalho de responder ao questionário, reduzindo o esforço físico e mental necessários e eliminando situações embaraçosas, implicações de subordinação e o desembolso de dinheiro;

- maximizar a recompensa para quem responder, por exemplo, agradecendo à participação com antecipação e indicando que o resultado da pesquisa será enviado;

- fornecer alguma demonstração que conduza à credibilidade no trabalho por parte do informante, como, por exemplo, indicando a entidade que está financiando o estudo.

Com base em Dillman ${ }^{6}$ e em Sudman e Bradburn ${ }^{7}$ o desenvolvimento do questionário deve obedecer a certas regras, tais como:

- utilizar o maior número possível de questões fechadas e/ou parcialmente fechadas;

- responder às seguintes perguntas relativas a cada item que o pesquisador considera importante para o estudo:

* serão as palavras uniformemente entendidas?
* as questões contêm abreviaturas ou frases não convencionais?

* as questōes são muito vagas, muito precisas ou tendenciosas?

* as perguntas são inconvenientes ou embaraçosas?

* as perguntas exigem muito do respondente?

* existem questōes duplas, ou seja, duas perguntas são combinadas em uma, exigindo uma única resposta do informante?

* existem questōes mutuamente exclusivas?

* está sendo exigido muito conhecimento do informante?

* a pergunta é tecnicamente acurada?

* podem as respostas ser comparadas com informações existentes?

* as perguntas são difíceis de serem entendidas?

- ordenar as questões obedecendo aos seguintes critérios:

* colocar as perguntas em ordem descendente de importância. A primeira questão deve ser claramente relacionada com o tópico do estudo, fácil de responder e de entender, neutra (isto é, aplicável para todos), e interessante;

* agrupar as questões de assuntos similares, e colocá-las dentro das áreas temáticas por tipo de questão;

* tirar partido de laços cognitivos que os respondentes provavelmente fazem entre os grupos de questōes, construindo, por exemplo, um sentido de fluxo e continuidade em todo o questionário;

* posicionar as questões mais desagrađáveis aos respondentes a pós aquelas consideradas mais agradáveis em cada área temática;
4. GOODE, W. J., HAT, P. K. Métodos em pesquisa social. São Paulo: Nacional, 1979 p. 219 .

5. DILLMAN, Don A. Mail and telephone surveys: the total design method. USA: John Wiley \& Sons, Inc., 1978, p.36-128.

6. Idem, ibidem.

7. SUDMAN, S., BRADBURN, N. M. Asking questions. London: Jossey-Bass, 1982, p. 41-226. 
* colocar as questões demográficas, sempre que possível, no final do questionário. Tais perguntas são referentes aos aspectos como idade, remuneração, status no emprego etc., de ordem pessoal que, muitas vezes, são consideradas desagradáveis pelos respondentes;

* usar o formato de folheto (booklet) visando a facilitar a leitura. A aparência do questionário é muito importante, devendo ser fácil de responder e profissionalmente impresso. Todas as questões, e também as subpartes do questionário, devem ser numeradas.

Sudman e Bradburn explicam também que, para se desenvolver um bom questionário, os seguintes tópicos devem ser observados:

" conter o impulso de escrever perguntas especificas até ter concluído as questões da pesquisa;

- escrever as questóes de pesquisa e tê-las sempre consigo enquanto está elaborando o questionário;

- cada vez que escrever uma pergunta, pensar: 'por que quero saber isto?' A resposta a esse questionamento deve ser de tal forma que ajude a responder às questões da pesquisa". ${ }^{8}$

O pré-teste do questionário é essencial, podendo ser realizado com três grupos de pessoas: colegas de trabalho e profissionais da área para que avaliem se está de acordo com os objetivos do estudo; usuários potenciais dos dados ou uma pequena amostra dos respondentes similar à do universo pesquisado. Terminada essa etapa, as questões onde não se obteve o tipo de informação solicitada deverão ser eliminadas. As perguntas em que os respondentes tiveram dificuldade de entendimento deverão ser revisadas. Depois, um novo estudo piloto deve ser realizado.

Vale destacar que um questionário bem escrito e apresentado poderá ser considerado insatisfatório, caso não obtenha as informações necessárias para alcançar os objetivos da pesquisa.
Um outro aspecto que deve ser salientado em relação a esse método de coleta de dados diz respeito à carta de apresentação que acompanha o questionário. Ela serve para introduzir a pesquisa e motivar o respondente a preencher $\mathrm{e}$ retornar o questionário ao remetente. Goode e Hatt ${ }^{9}$ apresentam as seguintes características de seu conteúdo: o patrocínio; por que o estudo?; por que o informante deveria se preocupar em responder?; as instruções de como preencher o questinário e garantia de anonimato.

Moser ressalta que entre os passos para aumentar o número de respostas estão: " $a$ inclusão de envelopes selados ou carta resposta comercial com porte pago, promessa de anonimato e confidencialidade, uma boa carta de apresentação e um questionário simples e não muito extenso." 10

Caso o número de respostas não seja
8. Idem, ibidem, p. 13-4.

9. GOODE, W. J., HATT, P. K. Op. cit., p. 128.

10. MOSER, C. A. Op. cit., 0.179. suficiente, uma segunda remessa do questionário pode ser enviada, visando a aumentar o número de respondentes. Em alguns casos, até uma terceira tentativa deve ser realizada, de mado que seja obtido o maior número de respostas possível.

Em síntese, o questionário enviado pelo correio como método de coletar dados apresenta vantagens e limitaçōes. Em relação à primeira, destacam-se a economia relativa e a rapidez como as mais importantes; em relação à segunda, está o problema do número de não respondentes. Entretanto, se o método for aplicado com todo cuidado e seguindo muitas das regras discutidas anteriormente, apresenta-se como uma alternativa de baixo custo para realização de pesquisas, especialmente na atual conjuntura brasileira, onde cada vez mais os recursos financeiros são rarefeitos. 


\section{PESQUISAS REALIZADAS COM SUCESSO USANDO A COLETA DE DADOS ATRAVÉS DO QUESTIONÁRIO REMETIOO PELO CORREIO}

A seguir, são apresentadas três pesquisas que usaram o método de questionário enviado pelo correio e obtiveram bons resultados.

A primeira é a tese de doutorado de Moraes, intitulada Organization, strategy, strategic management and performance. of Brazilian firms, apresentada na Universidade de Manchester, na Inglaterra, que analisou empresas dos setores siderúrgico, têxtil, de açúcar e álcool e de produtos farmacêuticos.

Foram enviados 606 questionários, devidamente pré-testados, para empresas selecionadas de Visão - Quem é quem na economia brasileira (números de 1981 a 1986). Apesar da limitação financeira, todo esforço foi feito para produzir a melhor qualidade de impressão. Cada questionário enviado foi colocado num envelope que possuía a etiqueta de endereçamento impressa por computador. Um envelope com endereçamento para resposta, embora sem selo, foi incluído na correspondência. A carta de apresentação, feita em uma página, em linguagem direta, enfatizava: que as respostas teriam tratamento confidencial, ou seja, as empresas seriam identificadas por um número código somente pelo pesquisador; a utilidade do estudo para o contexto brasileiro; a participação da empresa seria muito importante para o sucesso da pesquisa; que a cópia dos principais resultados seria enviada para a empresa. Num local apropriado (como na carta de

\begin{tabular}{|lccc|}
\hline \multicolumn{4}{|c|}{ Tabela 1: Respostas das empresas pesquisadas por tipo de indústria } \\
\hline Tipo de indústria & $\begin{array}{c}\text { Questionários } \\
\text { recebidos }\end{array}$ & População & (\%) \\
\hline Siderurgia & 22 & 46 & 47,8 \\
Produtos farmacêuticos & 20 & 74 & 27,0 \\
Têxtil & 54 & 278 & 19,0 \\
Álcool e açúcar & 31 & 208 & 14,9 \\
\hline Total & 127 & 606 & $\mathbf{2 0 , 9}$ \\
\hline Fonte: MORAES, Walter F. A. de Op. cit., p. 86 \\
\hline
\end{tabular}



apresentação, página de rosto do questionário e no envelope), as iniciais e os logotipos da UMIST (University of Manchester Institute of Science and Technology) e UFPE (Universidade Federal de Pernambuco) foram impressos. Para aumentar a taxa de resposta, tudo foi realizado para demonstrar o caráter profissional da pesquisa.

Depois de quatro semanas, uma carta lembrando a primeira foi enviada a todas as empresas que não haviam respondido ao questionário. Ao fim da décima semana, uma terceira carta foi enviada, agora com um questionário incluído, para 167 empresas, aleatoriamente selecionadas, visto que, em virtude da limi-
11. MORAES, Walter F. A . de. Organization strategy, strategic management and performance of Brazilian firms. England: University of Manchester Institute of Science and Technology -UMIST, 1988. p. 84-6 (Tese de Doutorado) 


\begin{tabular}{|c|c|c|c|c|c|c|c|c|c|}
\hline \multirow{3}{*}{$\begin{array}{l}\text { Número de } \\
\text { empregados } \\
\text { por empresa }\end{array}$} & \multirow{3}{*}{$\begin{array}{l}\text { Corres- } \\
\text { pondências } \\
\text { expedidas }\end{array}$} & \multicolumn{8}{|c|}{ Questionários recebidos } \\
\hline & & \multicolumn{2}{|c|}{$1^{\mathrm{a}}$ remessa } & \multicolumn{2}{|c|}{$2^{\mathrm{a}}$ remessa } & \multicolumn{2}{|c|}{$3^{\frac{a}{a}}$ remessa } & \multicolumn{2}{|c|}{ Total } \\
\hline & & $n^{0}$ & $\%$ & $n^{n}$ & $\%$ & $n^{2}$ & $\%$ & $n^{0}$ & $\%$ \\
\hline $1-39$ & 103 & 7 & 6,7 & 1 & 1,0 & 18 & 17,4 & 26 & 25,0 \\
\hline $40-49$ & 60 & 5 & 8,3 & 6 & 10,0 & 17 & 28,3 & 28 & 46,7 \\
\hline $50-99$ & 128 & 19 & 14,8 & 25 & 19,5 & 27 & 21,0 & 71 & 55,0 \\
\hline $100-199$ & 100 & 22 & 22,0 & 40 & 40,0 & - & - & 62 & 62,0 \\
\hline Total & 391 & 53 & 13,5 & 72 & 18,4 & 62 & 15,8 & 187 & 47,8 \\
\hline
\end{tabular}

Fonte: OLIVEIRA, L.M.B. de., COSTA, S.P.A. da Op. cit. p. 8.

Tabela 3: Respostas das empresas pesquisadas (200 ou mais empregados)

\begin{tabular}{|c|c|c|c|c|c|c|c|c|c|}
\hline \multirow{3}{*}{$\begin{array}{l}\text { Número de } \\
\text { empregados } \\
\text { por emprésa }\end{array}$} & \multirow{3}{*}{$\begin{array}{l}\text { Corres- } \\
\text { pondencias } \\
\text { expedidas }\end{array}$} & \multicolumn{8}{|c|}{ Questionários recebidos } \\
\hline & & \multicolumn{2}{|c|}{12 remessá } & \multicolumn{2}{|c|}{$2^{2}$ remessa } & \multicolumn{2}{|c|}{$3^{2}$ remessa } & \multicolumn{2}{|c|}{ Tolal } \\
\hline & & $n^{\theta}$ & $\%$ & $n^{2}$ & $\%$ & $n^{\underline{e}}$ & $\%$ & $n^{0}$ & $\%$ \\
\hline $200-299$ & 53 & 8 & 15,1 & 2 & 3,8 & 2 & 3,8 & 12 & 22,7 \\
\hline $300-499$ & 83 & 16 & 19,3 & 9 & 10,8 & 7 & 8,4 & 32 & 48,5 \\
\hline $500-700$ & 39 & 4 & 10,3 & 1 & 2,6 & 2 & 5,1 & 7 & 18,0 \\
\hline acima de 700 & 81 & 11 & 13,6 & 24 & 29,6 & - & - & 35 & 43,2 \\
\hline Total & 256 & 39 & 15,2 & 36 & 14,1 & 11 & 4,3 & 86 & 33,6 \\
\hline
\end{tabular}

Fonte; OLIVEIRA, L.M.B.; COSTA, S.P.A. da. Op. cit., p.9.

12. OLIVEIRA, L. M. B. de., COSTA, S. P. A. da. Conhecimentos, habilidades $\&$ atitudes necessários ao profissional de recursos humanos do estado de Pernambuco. CNPq, 1991, p.6-8. (Relatório de Pesquisa). respostas. Como resultado de todos esses procedimentos, 17 empresas solicitaram um novo questionário. Além disso, os responsáveis por algumas associações ou grupos de empresas foram também visitados objetivando a obtenção de apoio à pesquisa: Isso foi muito importante, especialmente nas indústrias siderúrgicas e de produtos farmacêuticos.

A figura 1 mostra a distribuição de freqüência do número de questionário obtido da pesquisa revelando o efeito das subseqüentes remessas de cartas, telex e telefonemas (ver figura 1).

Vale destacar que, dos 606 questionários enviados, foram recebidas 127 respostas, o que corresponde a uma taxa de retorno de $20,9 \%$. O número de respostas encontrase na tabela 1, de acordo com os tipos de indústria pesquisada (ver tabela 1).

A amostra obtida foi analisada e considerada representativa das empresas, classificadas em termos de número de empregados e volume de vendas. Dessa forma, a metodologia adotada para coletar os dados foi repleta de êxito.

Uma segunda pesquisa que também utilizou esse método de coleta de dados foi desenvolvida por Oliveira e Costa. ${ }^{12} \mathrm{O}$ estudo intitulado Conhecimento, habilidades e atitudes necessários ao profissional de recursos humanos do cstado de Pernambuco contou com o apoio financeiro do $\mathrm{CNPq}$ - Conselho de Desenvolvimento Científico e Tecnológico.

Os dados foram coletados utilizando-se dois tipos de questionário: um para empresas com menos de 200 empregados $c$ outro para aquelas que reuniam 200 ou mais empregados. Os questionários foram pré-testados em un grupo executivo de $\mathrm{RH}$. As dúvidas surgidas quanto ao seu preenchimento foram eliminadas e algumas alterações foram introduzidas.

Foram enviadas para a maioria das empresas três remessas de questionário, visando a aumentar o número de respostas. Além disso, foram dados telefonemas de reforço para diversas empresas. As tabelas 2 e 3 mostram o número de cartas expedidas e recebidas em termos dos dois segmentos analisados. Observa-se que $47,8 \%$ da amostra escolhida das empresas que apresentavam menos de $200 \mathrm{em}-$ pregados (ou seja, 187 empresas) retor- 
naram o questionário respondido. Além disso, 49 correspondências $(12,5 \%)$ foram enviadas de volta aos remetentes porque a empresa tinha se mudado ou fechado e ainda oito cartas $(2,1 \%)$ foram anuladas por conterem imprecisão nas respostas. Assim, 57 questionários (ou seja, $14,6 \%$ das correspondências enviadas) foram inutilizados.

Cerca de um terço $(33,6 \%)$ ou 86 empresas que reúnem 200 ou mais empregados responderam ao questionário. Ademais, 19 cartas $(7,4 \%)$ foram devolvidas aos remetentes sem resposta e uma correspondência $(0,4 \%)$ foi anulada por imprecisão nas respostas. Dessa forma, 20 questionários $(7,8 \%)$ foram inutilizados.

O número de cartas retornadas sem resposta aos remetentes foi maior entre as empresas que reúnem menos de 200 empregados, devido ao encerramento das atividades ou $\mathrm{mu}$ dança de endereço. Como se percebe, isso tende a acontecer com mais freqüência com as pequenas empresas.

Dessa forma, o trabalho foi desenvolvido baseado nas respostas de 187 empresas reunindo menos de 200 empregados e de 86 empresas que englobavam 200 ou mais empregados. As amostras foram consideradas significativas e representativas da população. Portanto, a metodologia utilizada para coletar os dados também obteve êxito.

A outra pesquisa que exemplifica a aplicação do método em estudo foi desenvolvida pela empresa de consultoria americana Tower Perrin, em 1991, com o apoio financeiro da IBM. O estudo é intitulado Priorities for competitive advantage. A 21st century vision: a worldwide human resource study. Nesse caso, em cada país foram identificados os maiores empregadores e enviada uma carta ao presidente-executivo e outra ao responsável pela área de recursos humanos em cada empresa. Segundo os autores do trabalho, alguns dos maiores empregadores de um país podem ter subsidiárias em outros. Assim, foi utilizada a estratégia de convidar dois executivos em cada companhia de cada país onde a empresa era considerada uma grande empregadora. Como resultado, algumas grandes empresas aplicaram o questionário em sete ou oito países. Além disso, algumas empresas solicitaram questionários adicionais para realizar pesquisa interna. entre os seus profissionais e gerentes de recursos humanos. ${ }^{13}$

For a m convidados também para participar do trabalho professores universitários e consultores. Nesse caso não foi limitado o número de participantes por instituição. A justificativa dos pesquisadores foi que o número de faculdades conceituadas envolvidas com a área de recursos humanos e firmas de consultoria é restrito. $\mathrm{E}$, ainda, porque é reconhecido a significante concentração de avançados conhecimentos nessas instituições. Assim, eles tenderam a abordar aqueles pro-
13. TOWERPERRIN CONSULTORES. Priorities for competitive advantage. A21stcentury vision: a worldwide human resource study. USA: IBM 1991, p. 80 (Relatório de Pesquisa) 
Tabela 4: Número de executivos participantes por país/região

\begin{tabular}{|llr}
\hline Megeláo & País & \\
América Latina & Argentina & 42 \\
& Brasil & 159 \\
& México & 67 \\
& França & 81 \\
Europa & Alemanha & 295 \\
& Itália & 212 \\
& Reino Unido & 261 \\
América do Norte & Canadá & 120 \\
& Estados Unidos & 1.174 \\
Pacífico/Ásia & Austrália & 94 \\
& Japão & 387 \\
Tolal & Coréia & 69 \\
\hline
\end{tabular}

Fonte: Priorities for compefitive advantage. A 21st century vision: a worldwide human resource study. Estudo da IBM conduzido pela Towers Perrin Consultores, 1991 , p. 84.

14. Idem, ibidem, p. 81.

15. Idem, ibidem, p. 2.

\section{CONCLUSÃO}

O método de coleta de dados através de questionário remetido pelo correio é compensador quando adequadamente utilizado. Como o pesquisador não está presente no momento em que o respondente está preenchendo o questionário, uma série de critérios deve ser levada em consideração visando ao perfeito entendimento daquilo que se deseja pesquisar.

O questionário deve ser elaborado com clareza e pré-testado, apresentar o máximo de questões fechadas e ser elaborado na forma de booklet. As perguntas devem ser colocadas em ordem descendente de importância e distribuídas em áreas temáticas por tipo de questão, sendo colocadas em primeiro lugar aquelas consideradas mais agradáveis.

De forma a não trazer despesa para o respondente, deve-se usar o sistema de carta comercial com porte pago. Deve-se também garantir absoluta confidencialidade das respostas. É muito importante uma carta de apresentação, indicando a instituição que está conduzindo e financiando o estudo para evidenciar a seriedade do trabalho.

Na maioria das vezes, uma segunda remessa de cartas deve ser enviada aos pesquisados, visando a aumentar o número de respondentes, contribuindo para que a amostra se torne de fato significativa e representativa da população.

A aplicação dessa metodologia traz substancial redução de custos, principalmente quando os respondentes estão geograficamente dispersos. Além disso, possibilita maior precisão das respostas, especialmente quando as questỗes podem causar algum embaraço ao informante. Ademais, permite ao pesquisado a possibilidade de consultar documentos e/ou outros setores da empresa, sem pressão e responder no todo ou em partes na hora de sua conveniência, sem a presença de estranhos.

Sendo o método aplicado de forma criteriosa, baseando-se nos pontos discutidos neste artigo, acredita-se que é grande a eficácia de sua utilização, desde que o objeto da pesquisa seja compatível com a sua aplicação. 